\title{
Surface modification of YS-20 with polydopamine for improving the tribological properties of polyimide composites
}

\author{
Liangfei WU ${ }^{1,2}$, Zhaozhu ZHANG ${ }^{1,2, *}$, Mingming YANG ${ }^{1}$, Junya YUAN ${ }^{1}$, Peilong LI $^{1,2}, Z_{\text {Zujun CHEN }}^{1, *}$, Xuehu MEN \\ ${ }^{I}$ State Key Laboratory of Solid Lubrication, Lanzhou Institute of Chemical Physics, Chinese Academy of Sciences, Lanzhou 730000, \\ China \\ ${ }^{2}$ Center of Materials Science and Optoelectronics Engineering, University of Chinese Academy of Sciences, Beijing 100049, China \\ ${ }^{3}$ School of Physical Science and Technology, Lanzhou University, Lanzhou 730000, China \\ Received: 17 August 2020 / Revised: 20 October 2020 / Accepted: 14 November 2020 \\ (C) The author(s) 2020.
}

\begin{abstract}
Recently, great effort has been devoted to prepare various reinforce fillers to improve polymer performances, but ignoring the importance of raw polymer powders which are indispensable parts of hot-pressed polymer composites. Herein, we engineer raw polyimide (PI) powders with the assistance of polydopamine (PDA) in aqueous solutions. After the modification, polymer powders change from hydrophobic to hydrophilic, which makes it is possible to further modification of polymer powders in liquid phase. During the curing process of modified polymer powders, the partial dehydration of the catechol groups and crosslinking of PDA via C-O-C bonds are confirmed. Based on the features of PDA, a non-destructive mixing method is utilized to realize homogeneous dispersion of multi-walled carbon nanotubes (MWCNTs) in polymer matrix. In comparison with ball milling method, this way can preserve the integrated innate structure of MWCNTs effectively. Besides, by taking full advantage of the reducing and metal-coordination capability of PDA, $\mathrm{Cu}^{2+}$ is successfully loaded onto the surfaces of polymer powders. The related characterizations demonstrate that $\mathrm{Cu}^{2+}$ in situ converts to metallic copper rather than copper oxide during the hot pressing process. The tribological properties of corresponding polymer composites are also studied. These results indicate that modifying polymer powders with PDA is multi-profit and presents practical application prospect.
\end{abstract}

Keywords: polydopamine (PDA); carbon nanotube; metallic copper; wear resistance

\section{Introduction}

Polymer composites are among the most rapidly growing classes of materials that have been designed for various industrial applications because of their superior properties, such as easy manufacturability, low density, corrosion resistance, and excellent specific strength. A lot of efforts have been devoted to obtain the composite with high comprehensive performances and the integration of nano-materials into the polymer matrix is a facile and effective route. For instance, Xiao et al. [1] prepared hollow boron nitride microbeads through a salt-temple method and epoxy composites exhibited outstanding thermal performance due to the interconnected networks. Zhang et al. [2] synthesized hydroxyapatite nanorods with controlled size and achieved better mechanical properties of polyurea by in situ polymerization. Min et al. [3] fabricated aminefunctionalized graphene nanosheets and the tribological performance of polyimide (PI) was successfully improved because of the covalent bonds between graphene nanosheets and PI. Despite the studies which utilize nano-materials to enhance the performances of polymer composites have received much attention, obtaining homogenous dispersion of nano-materials in polymer matrix is still a crucial step and full of challenges.

*Corresponding authors: Zhaozhu ZHANG, E-mail: zzzhang@licp.cas.cn; Zujun CHEN, E-mail: chenzj@licp.cas.cn 
There are many novelty ways to reduce abrasion of friction pairs [4, 5], particularly, avoiding thermal and stress concentration caused by nanoparticle agglomerations is important to reduce friction and wear when polymer composites are applied for tribology field. For the composites prepared by means of hot press molding technique, ball milling treatment of raw polymer powders, and reinforcing fillers is a common method to improve the dispersibility of nanoparticles, even though it may damage and chop the structure of nanoparticles to some extent [6]. Mixing raw polymer powders and reinforcing fillers in liquid phase is benign and alternative, but most of raw polymer powders are hydrophobic which restrict the use of water as a dispersion medium. Though hydrophilic modification of polymer powders is rarely reported, it is still a direction with application prospect.

Composites prepared by means of hot press molding technique generally relate to high curing temperature, for example it is $350{ }^{\circ} \mathrm{C}$ at least for PI. Under such condition, some metal ions will be oxidized to form corresponding metal oxides and then well-dispersed nanoparticles can be realized. Thus the performances of polymer can be improved owning to the incorporation of well-dispersed reinforcing fillers by this in situ synthetic method. However, the interaction between polymer powders and metal ions is limited due to the inertia feature of polymer powders. Moreover, hydrophobicity also makes it difficult to mix polymer powders with metal ion precursors in aqueous solution. In order to chelate the metal ions, carboxyl or polyhydroxyl structure is necessary and fortunately, these groups are also hydrophilic. Therefore, modifying polymer powder with carboxyl or polyhydroxyl structure is multi-profit and it is of practical significance to find such a simple but universal way.

Recently, inspired by mussel chemistry, biopolymer polydopamine (PDA) has been widely used as a versatile surface modification material due to its extraordinary wet-adhesion properties [7]. The molecules of PDA contain high concentrations of amine and catechol functional groups, which endow PDA with good hydrophilicity, firm adhesion, and the ability to chelate metal cations [8-10]. For polymer composites, the deposited PDA layer on the surfaces of reinforcing fillers commonly has two functions. On the one hand, the layer can act as a bridge to improve the interfacial bonding strength between filler and polymer matrix [11]. On the other hand, PDA coating is proven to be an efficient secondary reaction platform, which leads to tailoring of hybrid structure [12]. However, most of current works concentrate on preparing novelty fillers or modifying existing fillers to enhance hotpressed polymer composites. It is noteworthy that raw polymer powders are also vital parts of ultimate polymer composites. Thereby more attention toward the treatment of polymer powders is reasonable and necessary.

Herein, we engineered raw PI powders with the assistance of PDA in aqueous solutions. After the modification, polymer powders changed from hydrophobic to hydrophilic. Based on the features of PDA, a non-destructive method was explored to homogeneously mix multi-walled carbon nanotubes (MWCNTs) and PI powders by introducing MWCNTs into dopamine solution. Compared to ball milling method, this method was milder and more efficient to improve the dispersibility of nanoparticles in polymer matrix. Otherwise, PI powders loaded with copper ions on their surfaces were also received by the addition of copper chloride into the dopamine solution. Related characterization revealed that the copper ions were in situ converted to metallic copper rather than copper oxide during hot pressing process. The tribological properties of PI composites prepared with modified polymer powders were further studied and the experimental results indicated the great application value of this method.

\section{Experimental}

\subsection{Materials}

MWCNTs with an average length of 10-30 $\mu \mathrm{m}$ were supplied by Nanjing XFNANO Materials ech. Co. Ltd. Copper chloride dehydrate was purchased from Tianjin Fuchen Chemical Reagents Factory. 3-Hydroxytyramine hydrochloride (DA) was purchased from Shanghai Energy Chemical Company. Tris(hydroxymethyl) aminomethane (TRIS, 99\%) was provided by Kermel Chemical Reagent Plant. PI powders (YS-20) were used with particles size less than $75 \mu \mathrm{m}$. Water used throughout the experiment was deionized. 


\subsection{Preparation of modified PI powders}

Firstly, $0.4 \mathrm{~g}$ DA and $1 \mathrm{ml}$ Tris- $\mathrm{HCl}$ buffer solution were dissolved in $200 \mathrm{ml}$ of deionized water. Then $10 \mathrm{~g}$ YS-20 were added into the aqueous solution by continuous stirring for $24 \mathrm{~h}$. The suspension was filtered and washed several times with deionized water to remove unreacted DA. Finally, the obtained powders (PDA-YS20) were dried at $60^{\circ} \mathrm{C}$ for $12 \mathrm{~h}$. YS-20 loaded with MWCNTs and $\mathrm{Cu}^{2+}$ were also prepared by addition of $0.2 \mathrm{~g}$ MWCNTs and $0.3 \mathrm{~g}$ $\mathrm{CuCl}_{2} \cdot 2 \mathrm{H}_{2} \mathrm{O}$ into the DA solution, respectively.

\subsection{Preparation of PI composites}

The composites were prepared by means of hot press molding technique. Briefly, $4 \mathrm{~g}$ modified polyimide powders were compressed and heated to $350{ }^{\circ} \mathrm{C}$ in a mold with a dimensional of $2 \mathrm{~mm} \times 35 \mathrm{~mm} \times 40 \mathrm{~mm}$. The pressure was kept at $10 \mathrm{MPa}$ for $1.2 \mathrm{~h}$ and then the specimens were cooled down at room temperature naturally. Figure 1 presented the schematic diagram of an overall manufacturing procedure for making PI composites with various dopamine-modified YS-20.

\subsection{Tribological tests}

Friction and wear tests were carried out using a ballon-disk tribometer under the ambient temperature. The counterpart ball was GCr15 with a diameter of $6 \mathrm{~mm}$. The applied load was $20 \mathrm{~N}$ and the rotation speed was $300 \mathrm{r} / \mathrm{min}$. The continuous friction lasted for $2 \mathrm{~h}$ and each friction test was conducted three times.

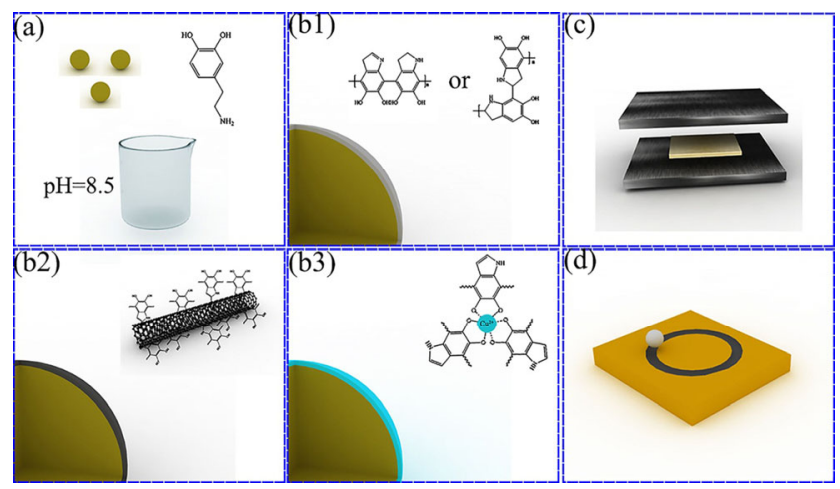

Fig. 1 The schematic diagrams of (a) YS-20 modified with PDA; (b1) PDA-YS20 and PDA-YS20 loaded with (b2) MWCNTs and (b3) $\mathrm{Cu}^{2+}$; (c) hot pressing of polymer powder; and (d) ballon-disk tribometer.

\subsection{Characterization}

A transmission electron microscope (TEM, Tecnai TF20) was used to visually observe the particle structure. The surface morphologies of nanoparticles and wear tracks were observed by a field-emission scanning electron microscope (FE-SEM, JSM-6701F, JEOL) and a scanning electron microscope (SEM, JSM-5600LV). Fourier transform infrared (FTIR) spectra were collected on a Bruker IFS 66v/s spectrometer. X-ray photoelectron spectroscopy (XPS) characterization was carried out on an ESCALAB 250xi spectrometer. Thermal analysis was performed on a thermo-gravimetric analysis instrument (TGA, STA 449 F3). Wear volumes of different wear tracks were measured by MicroXAM-3D noncontact surface mapping profiler. Ball milling of polymer powders were performed with GRINDER GT200. The duration is $2 \mathrm{~min}$ and the frequency was $1,000 \mathrm{rpm}$.

\section{Results and discussion}

\subsection{YS-20 modified with PDA}

The DA molecules were strongly oxidized in a basic environment and catechol was oxidized to benzoquinone, followed by the self-polymerization in a manner of melanin formation [13, 14]. PI powders were hydrophobic and most of them floated on water as shown in Fig. 2(a). After the deposition of PDA, the color of YS-20 altered from bright yellow to dark gray and PDA-YS20 displayed better compatibility with deionized water. Notably, this method not only improved the hydrophilicity of YS-20, but also applied to other common polymer powders, such as UHMWPE and PEEK powders (Fig. 2(a)). The FTIR spectra of asreceived YS-20 and PDA-YS20 were shown in Fig. 2(d) and both of them exhibited similar structure. Especially, the peaks located at around 1,778 and 1,715 $\mathrm{cm}^{-1}$ were the characteristics of $\mathrm{C}=\mathrm{O}$ asymmetric and symmetric peaks [15]. The absorption peaks of $\mathrm{C}-\mathrm{N}$ stretching vibration of imine ring located at $1,368 \mathrm{~cm}^{-1}$ [16]. The broad bands at $3,438 \mathrm{~cm}^{-1}$ resulted from the hydroxyl stretching vibrations [17]. In order to investigate the transformation of morphology and structure of PDA during hot pressing process, PDA particles were prepared without the addition of YS-20. The obtained 


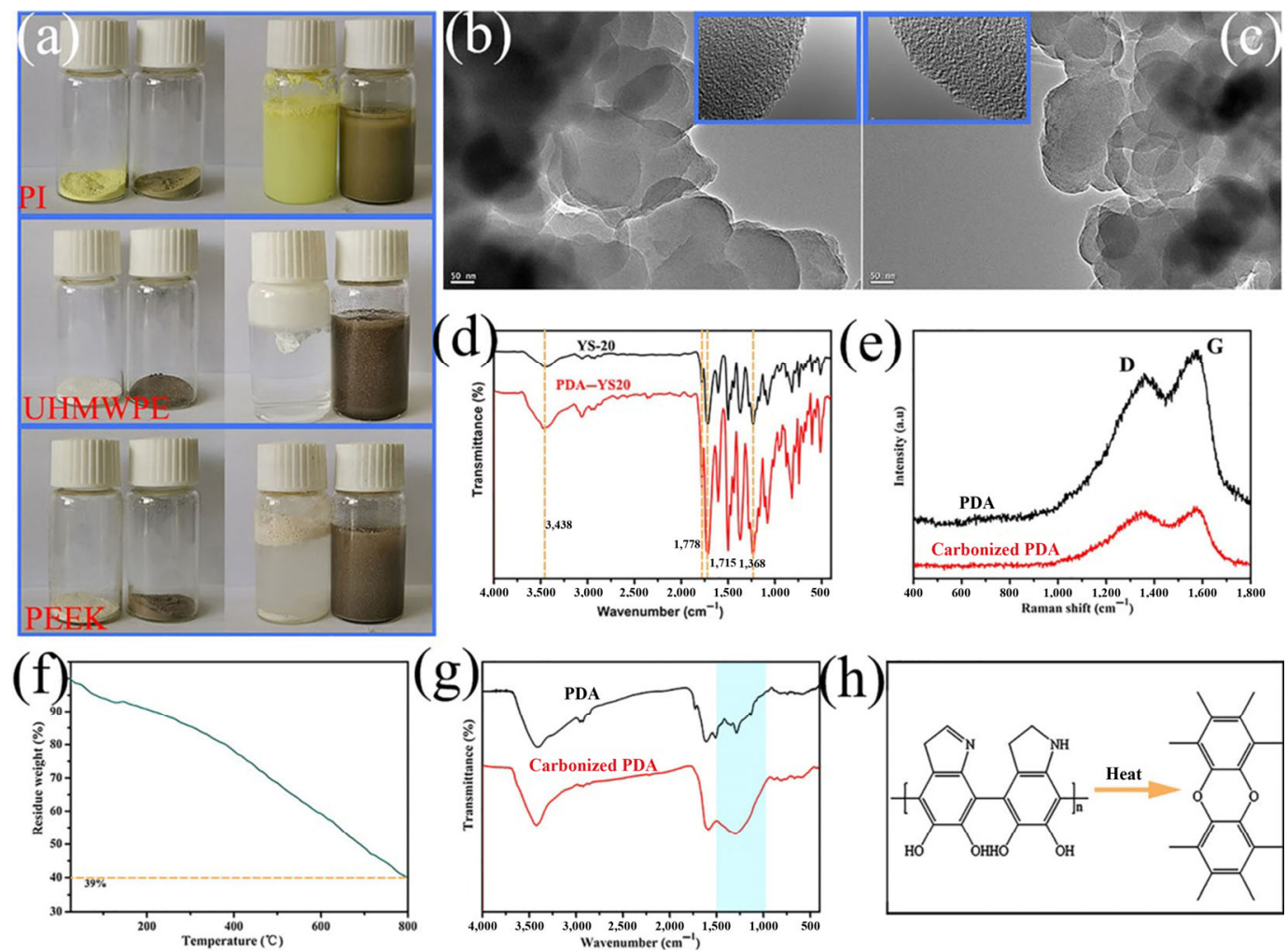

Fig. 2 (a) Digital pictures of different polymer powders in water; TEM and the magnified high resolution images (inset) of (b) PDA and (c) carbonized PDA; (d) FTIR spectra of YS-20 and PDA-YS20; (e) Raman spectra of PDA and carbonized PDA; (f) TGA result of PDA; (g) FTIR spectra of PDA and carbonized PDA; and (h) proposed chemical structural transformation of PDA during the curing process.

PDA particles were treated at high temperature by the same heating procedure which was used to prepare polymer composite under the protection of inert gas. TEM images indicated PDA particles were spherical and calcination had little effect on the morphologies due to lower heating rate (Figs. 2(b) and 2(c)). Yu et al. [18] reported the existence of distinct graphite-like nanostructure in the carbonized PDA particles but it was not observed in the magnified high-resolution image (Figs. 2(b) and 2(c)). One possible reason was that $350{ }^{\circ} \mathrm{C}$ was not enough to produce graphite-like structure. Figure 2(e) presented the Raman spectrum of PDA particles. Two dominating peaks appeared at 1,345 and 1,575 $\mathrm{cm}^{-1}$, corresponding to $\mathrm{D}$ band (catechol stretch) and G band (catechol deformation) [19]. TG analysis (Fig. 2(f)) showed the residue weight of PDA particles was about $80 \%$ with the temperature from 25 to $350{ }^{\circ} \mathrm{C}$ and it was about $40 \%$ when the temperature up to $800{ }^{\circ} \mathrm{C}$. The high residue weight implied the superior thermal stability of PDA particles. In the FTIR spectra (Fig. 2(g)), carbonized PDA showed a broad band in the range of $1,500-1,000 \mathrm{~cm}^{-1}$ compared to that of PDA particles. According to the work of Zou et al. [20], the broad band was mainly consisted of $\mathrm{C}-\mathrm{OH}, \mathrm{C}-\mathrm{O}$, and $\mathrm{C}-\mathrm{O}-\mathrm{C}$, indicating the partial dehydration of the catechol groups on adjacent PDA molecules [21]. Combined with the above analysis, it was speculated that the deposited PDA on the surfaces of YS-20 partially cross-linked via C-O-C bonds (Fig. 2(h)) and could maintain their original morphologies during the curing process. The degradation of some functional groups also existed.

The pure PI bulk materials prepared with YS-20 were orange and transparent while they were black and opaque when PDA-YS20 were used as the raw powders (pPI). Figure 3(a) displayed the fracture surfaces of PI and the composite. The pure PI matrix showed smooth and clean surfaces, which was attributed to brittle fracture. By contrast, the fractured surfaces of pPI became more rough (Fig. 3(d)), suggesting the incorporation of PDA could inhibit the crack propagation in polymer matrix [22]. Mean friction coefficient and wear volume of PI and pPI were displayed in Figs. 3(b) and 3(e). It was obvious that modifying YS-20 

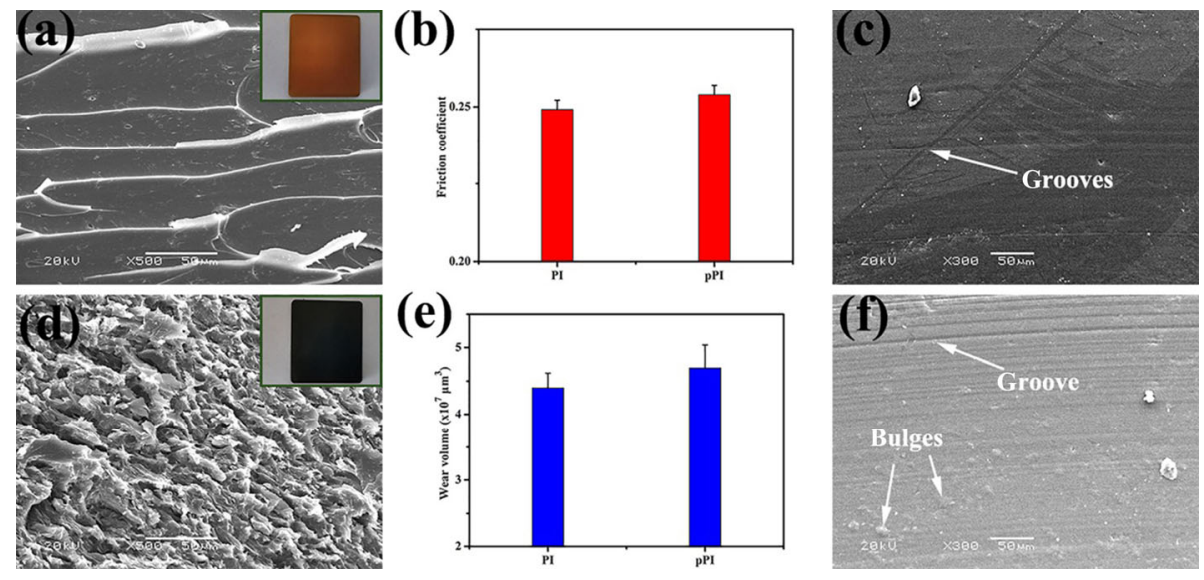

Fig. 3 The fracture surfaces of (a) PI and (d) pPI; (b) mean friction coefficient and (e) mean wear volume of PI and pPI; SEM micrographs of the wear tracks of (c) PI and (f) pPI.

with PDA was not good for improving the corresponding tribological properties. Figures $3(\mathrm{c})$ and $3(\mathrm{f})$ compared SEM micrographs of the wear tracks of PI and pPI. Deep grooves caused by the protruding asperities on the steel counterface [23], were observed on both wear tracks. Much pits and bulges came out on the wear tracks of pPI and the surfaces were coarser. To take full advantage of PDA and to enhance the tribological performances of PI composites, two facile and efficient improvements were made to PDA-YS20.

\subsection{PDA-YS20 loaded with MWCNTs (pCNT-YS20)}

MWCNTs were ideal reinforcements to develop high performance polymer composites owning to their excellent optical, thermal, and mechanical properties [24]. It was a consensus that the distribution state of MWCNTs had a great influence on the polymer properties [25]. Ball milling was a common mixing method, however, some issues such as flattening of polymer powders, shortening of MWCNTs length, and MWCNTs agglomeration have also raised $[6,26]$. In this work, when MWCNTs were put into the DA solution, the non-destructive modification of MWCNTs and homogeneous mixing of MWCNTs with YS-20 were received simultaneously by continuous stirring. YS-20 loaded with MWCNTs were also obtained by ball milling method and they were named as mCNT-YS20. Figures 4(a)-4(d) showed the surface morphologies of pCNT-YS20 and mCNT-YS20. It was found that the surfaces of YS-20 were rough and irregular. For mCNT-YS20, one part of the MWCNTs was distributed relatively uniform on the surfaces of

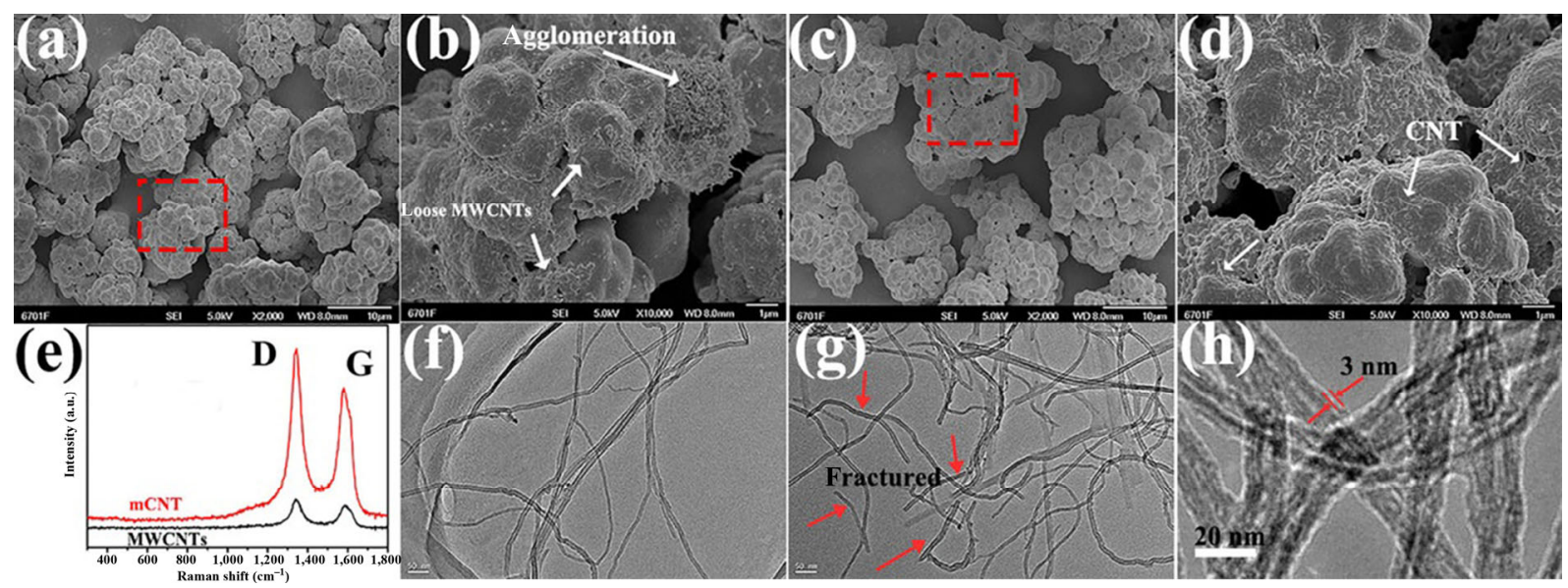

Fig. 4 The surface morphologies of (a, b) mCNT-YS20 and (c, d) pCNT-YS20; (e) Raman spectra of MWCNTs and mCNT; TEM images of (f) pristine MWCNTs, (g) mCNT, and (h) PDA-CNT. 
YS-20, while the other part was agglomerated. For pCNT-YS20, the agglomeration has been alleviated and most MWCNTs were tightly bound to YS-20. Tight binding represented better interfacial affinity between MWCNTs and polymer powders, thus the stress transfer from polymer matrix to reinforcement fillers in cured polymer was strengthened followed by enhanced mechanical and tribological performances of polymer composites. Raman spectroscopy was a widely used method to determine the defect structures of carbon-based materials. In Fig. 4(e), two remarkable bands at 1,345 and 1,586 $\mathrm{cm}^{-1}$ could be observed, which was resulted from the D-band and G-band of graphitic carbon [27]. The intensity ratio of D-band to G-band $\left(I_{\mathrm{D}} / I_{\mathrm{G}}\right)$ was employed to assess the degree of structural defects in pristine MWCNTs and ball-milled MWCNTs (mCNT). The $I_{\mathrm{D}} / I_{\mathrm{G}}$ ratio slightly shifted from 1.19 for pristine MWCNTs to 1.27 for $\mathrm{mCNT}$, indicating the partially damaged structure after ball milling. The Raman peaks of MWCNTs and PDA particles overlapped to some extent, so the Raman spectra was not a true reflection of the defects of PDA modified MWCNT (pCNT) and it was not supplied here. From the TEM images in Figs. 4(f)-4(h), it was obvious that the pristine MWCNTs possessed tubular and curving structure with an average diameter about $10 \mathrm{~nm}$. After the ball milling, pristine MWCNTs fractured and their length decreased marked by the red arrows in Fig. 4(g), which was consistent with higher ratio of $I_{\mathrm{D}} / I_{\mathrm{G}}$. As shown in Fig. $4(\mathrm{~h})$, the surfaces of $\mathrm{pCNT}$ were wrapped with a $3 \mathrm{~nm}$ thick continuous amorphous layer, demonstrating a successful deposition of PDA.

Figures 5(a) and 5(b) depicted the mean friction coefficient and wear volume of PI, PI composites prepared with mCNT-YS20 (mCNT-PI), and PI composites prepared with pCNT-YS20 (pCNT-PI). It was obvious the wear resistance of mCNT-PI and pCNT-PI is both better than that of pure PI, which confirmed the positive wear reducing effect of MWCNTs. As to the worn surface of mCNT-PI (Fig. 5(c)), there were apparent furrows and holes. The stress bearing capacity of MWCNTs became weaker since their integrated innate structure was destroyed by ball milling. Otherwise, ball milling reduce the aspect ratio of MWCNTs, which impeded the formation of percolated network in polymer matrix and decreased the interfacial bonding [28]. Thus the steel counterface penetrated the polymer matrix more easily and more scratches appeared on the worn surface. Besides, the friction stress was mainly concentrated at the matrix-filler
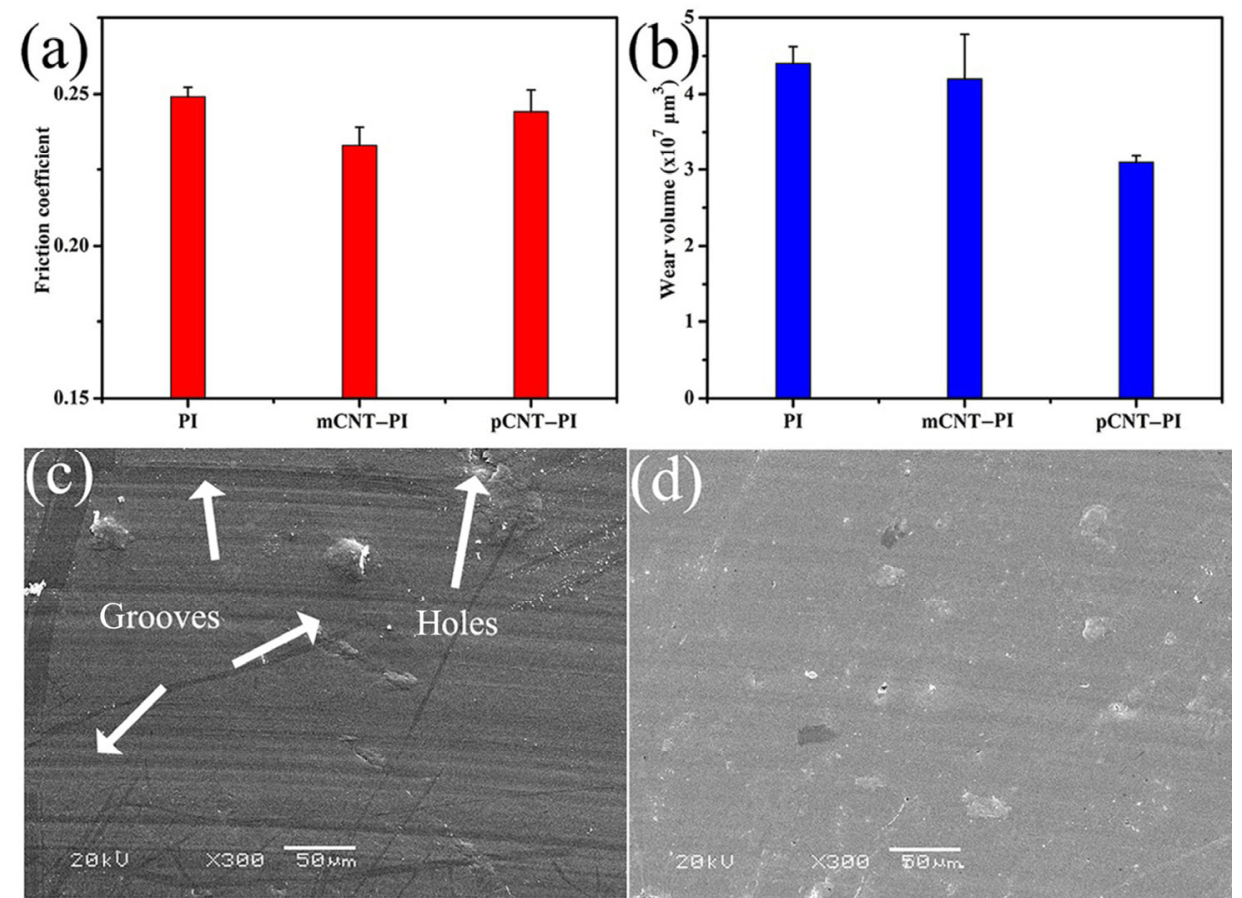

Fig. 5 (a) Mean friction coefficient and (b) mean wear volume of PI, mCNT-PI, and pCNT-PI; SEM micrographs of the wear tracks of (c) mCNT-PI and (d) pCNT-PI. 
interface due to the superior mechanical strength of MWCNTs. Micro-cracks propagated along the agglomeration of MWCNTs, resulting in a large volume loss on the worn surface [29]. For the worn surface of pCNT-PI (Fig. 5(d)), it was much smoother and flatter. The non-destructive mixing method not only preserved the strength of MWCNTs, but also improved their dispersion state in polymer matrix, thus a better tribological performance of polymer composites was achieved.

\subsection{PDA-YS20 loaded with $\mathrm{Cu}^{2+}(\mathrm{pCu}-\mathrm{YS} 20)$}

Bio-inspired catechol-based compounds were typical organic ligand for metal-coordination [30]. Thus a co-deposition strategy could be realized by the addition of $\mathrm{Cu}^{2+}$ into the DA solution. During the continuous stirring, DA chelated $\mathrm{Cu}^{2+}$ to self-polymerize and deposited onto the surfaces of YS-20 at the same time. The element mapping of modified PDA-YS20 (Fig. 6(a1)) indicated the relatively homogeneous dispersion of $\mathrm{Cu}^{2+}$. Similarly, this loading method was applicable to other metal cations, such as $\mathrm{Ni}^{2+}$ and $\mathrm{Zn}^{2+}$ (Figs. 6(a2) and 6(a3)). XPS was performed to identify the surface chemical valence state of $\mathrm{Cu}^{2+}$ before and after hot pressing process. As elucidated in Figs. 6(b) and 6(c), the high-resolution $\mathrm{Cu} 2 \mathrm{p}$ spectrum of PDA after the incorporation of $\mathrm{Cu}^{2+}(\mathrm{PDA}-\mathrm{Cu})$ displayed two distinct signals for $\mathrm{Cu} 2 \mathrm{p}_{1 / 2}$ and $\mathrm{Cu} 2 \mathrm{p}_{3 / 2}$. The binding energy at 952.4 and $932.4 \mathrm{eV}$ were assigned to zero valent copper $\left(\mathrm{Cu}^{0}\right)$, while the $\mathrm{Cu}$ $2 p_{1 / 2}$ at $954.6 \mathrm{eV}$ and $\mathrm{Cu} 2 \mathrm{p}_{3 / 2}$ at $934.7 \mathrm{eV}$ were attributed to $\mathrm{Cu}^{2+}[31,32]$. The existence of $\mathrm{Cu}^{0}$ was related to the strong reducing capability of PDA, which contained many catechol moieties and secondary amino groups $[12,33]$. The satellite peaks at 963 and $944 \mathrm{eV}$ was because of the open-shell $3 \mathrm{~d}$ character of $\mathrm{Cu}^{2+}$ [34]. After the carbonization, the peak intensity of $\mathrm{Cu}^{2+}$ weakened, suggesting high temperature could enhance the reducibility of PDA and more $\mathrm{Cu}^{2+}$ could convert into metallic copper. The residual satellite peaks in Fig. 6(c) were might due to the surface oxidation of copper when the sample was exposed to air. The XRD patterns of PI composites were investigated to further reveal structural changes of the $\mathrm{Cu}^{2+}$ after curing. In Figs. 6(e) and 6(f), both PI and pPI showed broad diffraction peaks at $\sim 22^{\circ}$ while a new weak peak appeared at $43.2^{\circ}$ for PI that prepared with $\mathrm{pCu}-$ YS20. The peak corresponded to the (111) of metallic copper, also indicating the existence of $\mathrm{Cu}^{0}$ [35]. Details of morphology evolution of PDA-Cu were characterized by TEM. In Fig. 7(a), the boundaries of PDA particles disappeared and more PDA particles connected together in a sheet-like structure. Du et al. [36] pointed out that adhesion ability of PDA could be significantly enhanced after coordination. Yang et al. [37] suggested that metal ions would complex with dopamine oligomers and produce stacked aggregates by coordination chemistry. These phenomena could be indirectly reflected in Fig. 6(d), the PDA were fluffy after freeze drying, while PDA-Cu easily assembled into huge sticky aggregates. In high-resolution TEM images (Fig. 7(b)), the crystal lattice fringes with
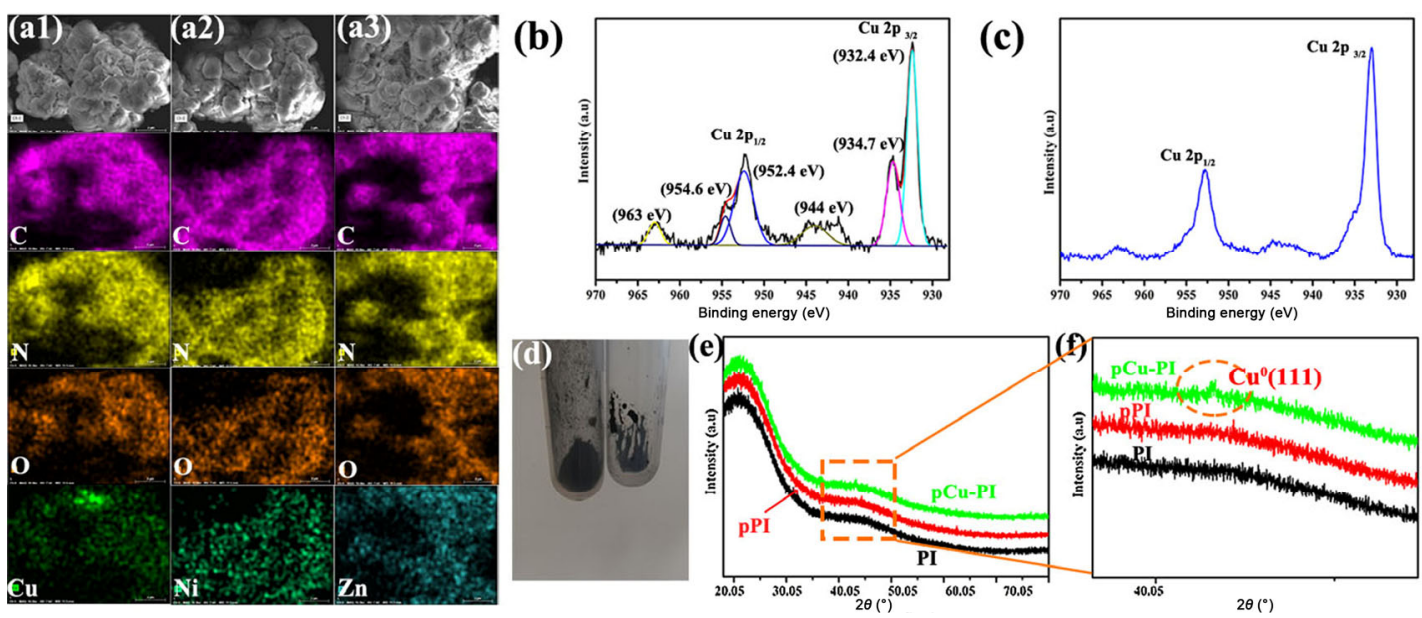

Fig. 6 (a1-a3) The element mapping of YS-20 loaded with $\mathrm{Cu}^{2+}, \mathrm{Ni}^{2+}$, and $\mathrm{Zn}^{2+}$; XPS spectra of (b) PDA-Cu and (c) carbonized PDA-Cu; (d) digital pictures of PDA and PDA-Cu after freeze drying; (e, f) XRD patterns of PI, pPI, and pCu-PI. 


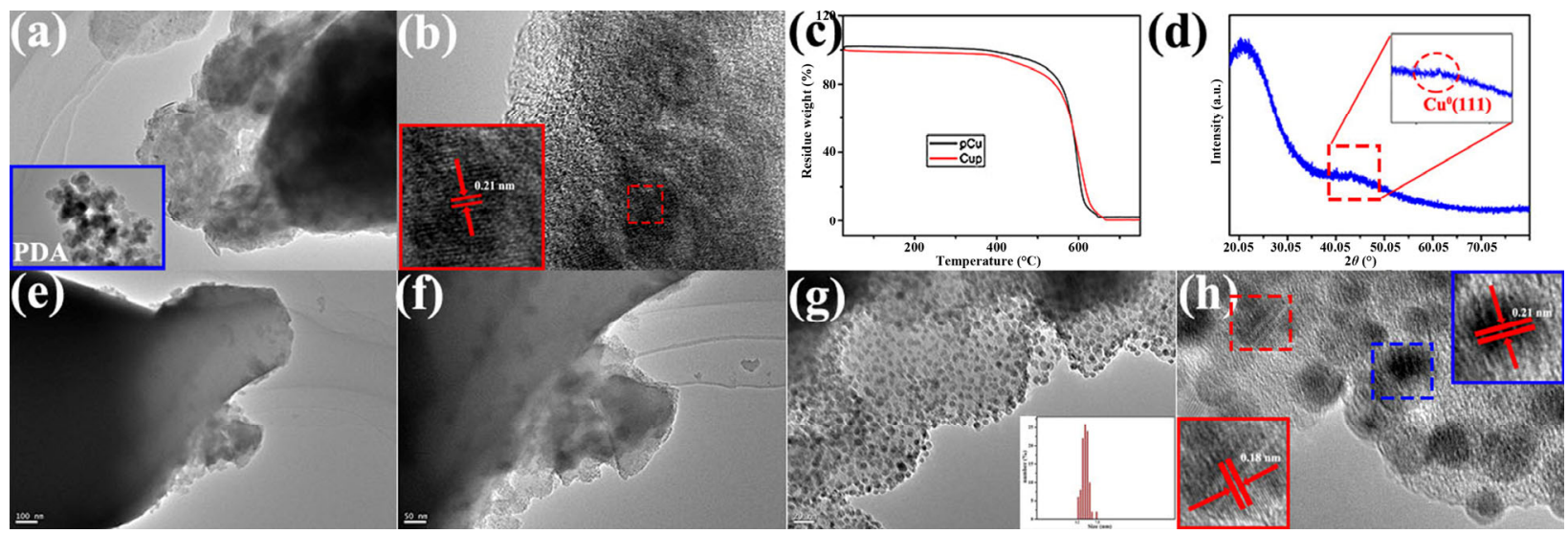

Fig. 7 TEM images of (a, b) PDA-Cu and (e-h) carbonized PDA-Cu; (c) TGA results of pCu-YS20 and p-Cu-YS20; (d) XRD patterns of $\mathrm{p}-\mathrm{Cu}-\mathrm{PI}$.

spacing of $0.21 \mathrm{~nm}$ corresponded to the (111) planes of metallic copper [38]. After carbonized, more metallic copper nanoparticles with high dispersity were observed and the particle sizes were about $5.42 \mathrm{~nm}$. The excellent particle uniformity was because of this in situ synthetic method. In Fig. 7(f), it was clearly recognized two distinct lattice fringes and the lattice plane spacing was about 0.18 and $0.21 \mathrm{~nm}$, which was in agreement with (200) and (111) planes of metallic copper, respectively [39]. The analysis described above demonstrated that $\mathrm{Cu}^{2+}$ in situ converted to metallic copper rather than copper oxide during the hot pressing process and a well-dispersed state of reinforcing filler was obtained.

There were two ways to prepare YS-20 particles loaded with $\mathrm{Cu}^{2+}$. The one way was to stir YS-20 in the mixed solution of DA and $\mathrm{Cu}^{2+}$. The other way was to stir PDA-YS20 in the solution of $\mathrm{Cu}^{2+}(\mathrm{p}-\mathrm{Cu}-\mathrm{YS} 20)$. TG analysis of two different YS-20 particles was performed in Fig. 7(c). The results confirmed residue weight of $\mathrm{pCu}-\mathrm{YS} 20$ was higher than that of $\mathrm{p}-\mathrm{Cu}-$ YS20, implying more $\mathrm{Cu}^{2+}$ loading in $\mathrm{pCu}-\mathrm{YS} 20$. The XRD pattern of $\mathrm{p}-\mathrm{Cu}-\mathrm{PI}$ also showed a peak at $43.2^{\circ}$, indicating the formation of $\mathrm{Cu}^{0}$ (Fig. 7(d)). Figures 8(a) and $8(\mathrm{~b})$ summarized the mean friction coefficient and wear volume of PI, pCu-PI, and p-Cu-PI. The friction coefficient of the three PI composites remained basically unchanged, but the wear volume reduced by $27 \%$ for p-Cu-PI and $36 \%$ for pCu-PI. In Figs. 8(c) and $8(\mathrm{~d})$, the furrow effect was suppressed for both
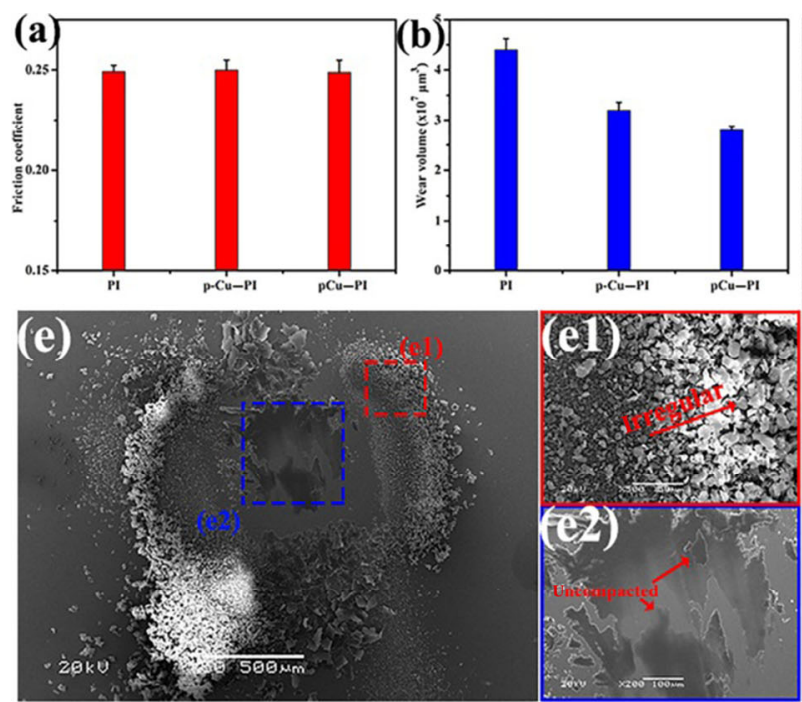

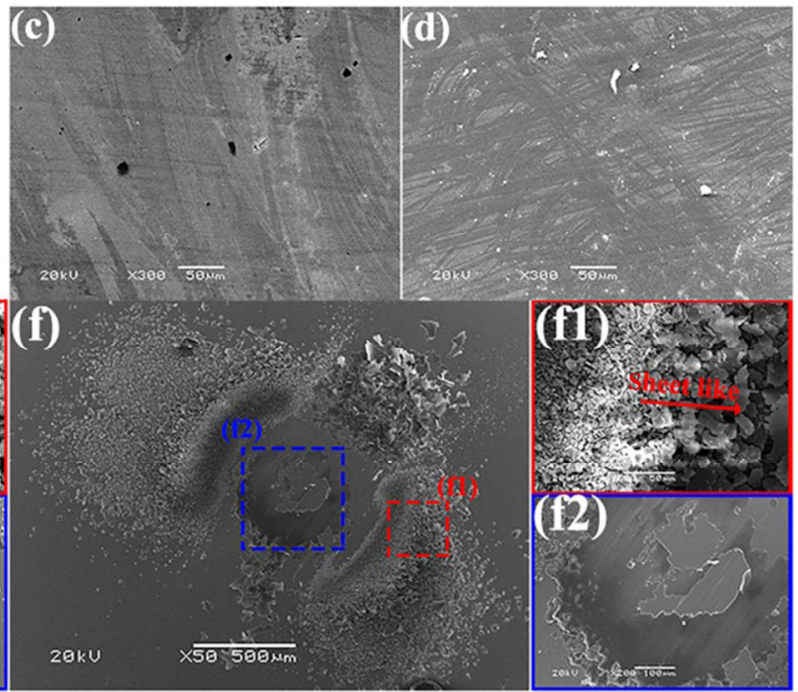

Fig. 8 Mean (a) friction coefficient and (b) wear volume of PI, p-Cu-PI, and pCu-PI; SEM micrographs of the wear tracks of (c) pCu-PI, (d) p-Cu-PI, and counterpart balls of (e) PI, (f) pCu-PI; locally amplification of counterpart balls of (e1, e2) PI and (f1, f2) pCu-PI. 
of $\mathrm{p}-\mathrm{Cu}-\mathrm{PI}$ and $\mathrm{pCu}-\mathrm{PI}$ and the worn surfaces were smooth in comparison with that of pure PI. The SEM images of worn surfaces of the counterpart balls were shown in Figs. 8(e) and 8(f) and patch-like tribofilms were formed for both PI composites. For pure PI, the wear debris (labeled by red arrow in Fig. 8(e1)) was irregular with different thickness and some uncompacted wear debris was found on the transfer film (Fig. 8(e2)). The uncompacted wear debris increased the roughness of transfer film and was easy to fall off from the counterpart ball, which would aggravate abrasive wear. For pCu-PI, the wear debris was sheet like (Fig. 8(f1)) and the transfer film was clean as well as compacted (Fig. 8(f2)). The successful load of $\mathrm{Cu}^{2+}$ and the conversion from $\mathrm{Cu}^{2+}$ to metallic copper contributed to strengthen the tribofilm, thus the wear resistance of PI composites could be reinforced.

\section{Conclusions}

In summary, we engineered YS-20 with the assistance of PDA in aqueous solutions. After the modification, polymer powders changed from hydrophobic to hydrophilic, which make it was possible to further modify polymer powders in liquid phase. During the curing process, the partial dehydration of the catechol groups and crosslinking of PDA via $\mathrm{C}-\mathrm{O}-\mathrm{C}$ bonds were confirmed from our experimental results. By taking full advantage of PDA features, two different manners were developed to improve the tribological properties of PI composites. The one way was to add MWCNTs into the DA solution, which could achieve non-destructive and homogeneous dispersion of MWCNTs in polymer matrix. The other way was to add $\mathrm{Cu}^{2+}$ into the DA solution, which in situ converted to metallic copper rather than copper oxide during the hot pressing process owning to the reducing capability of PDA. These two efficient and simple ways indicated that modifying polymer powders with PDA was multiprofit and presented practical application prospect.

\section{Acknowledgements}

The authors gratefully acknowledge the financial support from the National Natural Science Foundation of China (Grant Nos. 51805516 and 51675252) and the CAS 'Light of West China' program.
Open Access This article is licensed under a Creative Commons Attribution 4.0 International License, which permits use, sharing, adaptation, distribution and reproduction in any medium or format, as long as you give appropriate credit to the original author(s) and the source, provide a link to the Creative Commons licence, and indicate if changes were made.

The images or other third party material in this article are included in the article's Creative Commons licence, unless indicated otherwise in a credit line to the material. If material is not included in the article's Creative Commons licence and your intended use is not permitted by statutory regulation or exceeds the permitted use, you will need to obtain permission directly from the copyright holder.

To view a copy of this licence, visit http://creativecommons.org/ licenses/by/4.0/.

\section{References}

[1] Xiao C, Tang Y, Chen L, Zhang X, Zheng K, Tian X. Preparation of highly thermally conductive epoxy resin composites via hollow boron nitride microbeads with segregated structure. Compos Part A 121: 330-340 (2019)

[2] Zhang T, Cai W, Chu F, Zhou F, Liang S, Ma C, Hu Y. Hydroxyapatite/polyurea nanocomposite: Preparation and multiple performance enhancements. Compos Part A 128: 105681 (2020)

[3] Min C, Liu D, Qian J, He Z, Jia W, Song H, Guo L. High mechanical and tribological performance polyimide nanocomposites using amine-functionalized graphene nanosheets. Tribol Int 131: 1-10 (2019)

[4] Huang J, Li Y, Jia X, Song H. Preparation and tribological properties of core-shell $\mathrm{Fe}_{3} \mathrm{O}_{4} @ \mathrm{C}$ microspheres. Tribol Int 129: 427-435 (2019)

[5] Wang W, He Y, Zhao J, Mao J, Hu Y, Luo J. Optimization of groove texture profile to improve hydrodynamic lubrication performance: Theory and experiments. Friction 8: 83-94 (2020)

[6] Baena J.-C, Peng Z. Dispersion state of multi-walled carbon nanotubes in the UHMWPE matrix: Effects on the tribological and mechanical response. Polym Test 71: 125-136 (2018)

[7] Yang D, Ni Y, Kong X, Gao D, Wang Y, Hu T, Zhang L. Mussel-inspired modification of boron nitride for natural rubber composites with high thermal conductivity and low dielectric constant. Compo Sci Technol 177: 18-25 (2019)

[8] Li X, Gao P, Tan J, Xiong K, Maitz M F, Pan C, Wu H, Chen Y, Yang Z, Huang N. Assembly of metal-phenolic/ 
catecholamine networks for synergistically anti-inflammatory, antimicrobial, and anticoagulant coatings. ACS Appl Mater Interfaces 10: 40844-40853 (2018)

[9] Wu X, Jiang Q, Ghim D, Singamaneni S, Jun Y S. Localized heating with a photothermal polydopamine coating facilitates a novel membrane distillation process. J Mater Chem A 6 : 18799-18807 (2018)

[10] Wang Z G, Yang Y L, Zheng Z L, Lan R T, Dai K, Xu L, Huang H D, Tang J H, Xu J Z, Li Z M. Achieving excellent thermally conductive and electromagnetic shielding performance by nondestructive functionalization and oriented arrangement of carbon nanotubes in composite films. Compos Sci Technol 194: 108190 (2020)

[11] Shanmugam L, Feng X, Yang J. Enhanced interphase between thermoplastic matrix and UHMWPE fiber sized with CNT-modified polydopamine coating. Compos Sci Technol 174: 212-220 (2019)

[12] Wang Z, Yang M, Cheng Y, Liu J, Xiao B, Chen S, Huang J, Xie Q, Wu G, Wu H. Dielectric properties and thermal conductivity of epoxy composites using quantum-sized silver decorated core/shell structured alumina/polydopamine. Compos Part A 118: 302-311 (2019)

[13] Liu Y, Wu K, Luo F, Lu M, Xiao F, Du X, Zhang S, Liang L, Lu M. Significantly enhanced thermal conductivity in polyvinyl alcohol composites enabled by dopamine modified graphene nanoplatelets. Compos Part A 117: 134-143 (2019)

[14] Yuan J, Zhang Z, Yang M, Guo F, Men X, Liu W. Carbon nanotubes coated hybrid-fabric composites with enhanced mechanical and thermal properties for tribological applications. Compos Part A 102: 243-252 (2017)

[15] Zhu L, You L, Shi Z, Song H, Li S. An investigation on the graphitic carbon nitride reinforced polyimide composite and evaluation of its tribological properties. J Appl Polym Sci 134: 45403 (2017)

[16] Zhang D, Wang C, Wang Q, Wang T. High thermal stability and wear resistance of porous thermosetting heterocyclic polyimide impregnated with silicone oil. Tribol Int 140: 105728 (2019)

[17] Zhu Z, Yao H, Dong J, Qian Z, Dong W, Long D. Highmechanical-strength polyimide aerogels crosslinked with 4,4'-oxydianiline-functionalized carbon nanotubes. Carbon 144: 24-31 (2019)

[18] Yu X, Fan H, Liu Y, Shi Z, Jin Z. Characterization of carbonized polydopamine nanoparticles suggests ordered supramolecular structure of polydopamine. Langmuir 30: 5497-5505 (2014)

[19] Wang H, Lin C, Zhang X, Lin K, Wang X, Shen S. G. Musselinspired polydopamine coating: A general strategy to enhance osteogenic differentiation and osseointegration for diverse implants. ACS Appl Mater Interfaces 11: 7615-7625 (2019)

[20] Zou R, Liu F, Hu N, Ning H, Jiang X, Xu C, Fu S, Li Y, Zhou X, Yan C. Carbonized polydopamine nanoparticle reinforced graphene films with superior thermal conductivity. Carbon 149: 173-180 (2019)

[21] Kong J, Yee W. A, Yang L, Wei Y, Phua S. L, Ong H. G, Ang J. M, Li X, Lu X. Highly electrically conductive layered carbon derived from polydopamine and its functions in $\mathrm{SnO}_{2}$-based lithium ion battery anodes. Chem Commun 48: 10316-10318 (2012)

[22] Chen J, Luo K, Zhu J, Yu J, Wang Y, Hu Z. Reversibly cross-linked fullerene/polyamide composites based on DielsAlder reaction. Compos Sci Technol 176: 9-16 (2019)

[23] Fan X, Li G, Guo Y, Zhang L, Xu Y, Zhao F, Zhang G. Role of reinforcement types and silica nanoparticles on tribofilm growth at PTFE-steel interface. Tribol Int 143: 106035 (2020)

[24] Yang M, Yuan J, Guo F, Wang K, Zhang Z, Men X, Liu W. A biomimetic approach to improving tribological properties of hybrid PTFE/Nomex fabric/phenolic composites. Eur Polym J 78: 163-172 (2016)

[25] Kharitonov A P, Maksimkin A V, Mostovaya K S, Kaloshkin S D, Gorshenkov M V, D'Yachkova T P, Tkachev A G, Alekseiko L N. Reinforcement of bulk ultrahigh molecular weight polyethylene by fluorinated carbon nanotubes insertion followed by hot pressing and orientation stretching. Compos Sci Technol 120: 26-31 (2015)

[26] Enqvist E, Ramanenka D, Marques P A A P, Gracio J, Emami N. The effect of ball milling time and rotational speed on ultra high molecular weight polyethylene reinforced with multiwalled carbon nanotubes. Polym Compos 37: 1128-1136 (2016)

[27] Wang Y Y, Zhou Z H, Zhou C G, Sun W J, Gao J F, Dai K, Yan D X, Li Z M. Lightweight and robust carbon nanotube/ polyimide foam for efficient and heat-resistant electromagnetic interference shielding and microwave absorption. ACS Appl Mater Interfaces 12: 8704-8712 (2020)

[28] Manoj Kumar R, Sharma S. K, Manoj Kumar B. V, Lahiri D. Effects of carbon nanotube aspect ratio on strengthening and tribological behavior of ultra high molecular weight polyethylene composite. Compos Part A 76: 62-72 (2015)

[29] Lee J. H, Park M S, Lee C S, Han T S, Kim J H. Wearresistant carbon nanorod-embedded poly(vinylidene fluoride) composites with excellent tribological performance. Compos Part A 129: 105721 (2020)

[30] Zhao J, Fu N, Liu R. Graphite-wrapped Fe core-shell nanoparticles anchored on graphene as pH-universal electrocatalyst for oxygen reduction reaction. ACS Appl Mater Interfaces 10: 28509-28516 (2018) 
[31] Wu H, Li H, Zhao X, Liu Q, Wang J, Xiao J, Xie S, Si R, Yang F, Miao S, Guo X, Wang G, Bao X. Highly doped and exposed $\mathrm{Cu}(\mathrm{i})-\mathrm{N}$ active sites within graphene towards efficient oxygen reduction for zinc-air batteries. Energy Environ Sci 9: 3736-3745 (2016)

[32] Wen X, Qi H, Cheng Y, Zhang Q, Hou C, Guan J. Cu nanoparticles embedded in $\mathrm{N}$-doped carbon materials for oxygen reduction reaction. Chin J Chem 38: 941-946 (2020)

[33] Zhang L, Liu Z, Liu L Y, Ju X J, Wang W, Xie R, Chu L Y. Novel smart microreactors equipped with responsive catalytic nanoparticles on microchannels. ACS Appl Mater Interfaces 9: 33137-33148 (2017)

[34] Liu Y, Yang Y, Sun Q, Wang Z, Huang B, Dai Y, Qin X, Zhang X. Chemical adsorption enhanced $\mathrm{CO}_{2}$ capture and photoreduction over a copper porphyrin based metal organic framework. ACS Appl Mater Interfaces 5: 7654-7658 (2013)

[35] Xie Y, Zhang C, He X, Su J W, Parker T, White T, Griep M, Lin J. Copper-promoted nitrogen-doped carbon derived from zeolitic imidazole frameworks for oxygen reduction reaction. Appl Surf Sci 464: 344-350 (2019)

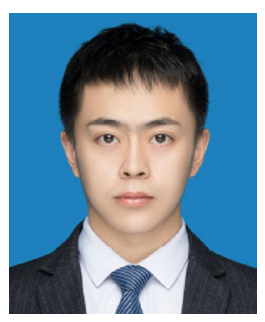

Liangfei WU. He received his bachelor degree in applied chemistry in 2016 from Anhui University, Hefei, China. After then, he was a Ph.D. student in the State Key Laboratory

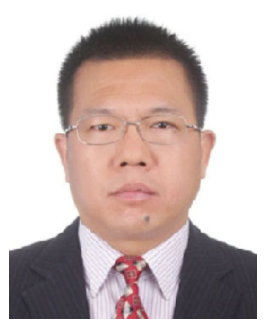

Zhaozhu ZHANG. He received his Ph.D. degree from Lanzhou Institute of Chemical Physics in 1998. His current research interests cover the tribology of composite materials,

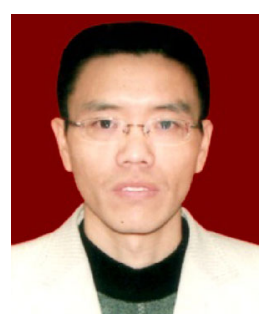

Zujun CHEN. He received his bachelor degree in materials science and engineering in 1999 from Nanchang Hangkong University. After
[36] Du C, Li M, Cao M, Song S, Feng S, Li X, Guo H, Li B. Mussel-inspired and magnetic Co-functionalization of hexagonal boron nitride in poly(vinylidene fluoride) composites toward enhanced thermal and mechanical performance for heat exchangers. ACS Appl Mater Interfaces 10: 34674-34682 (2018)

[37] Yang L, Kong J, Zhou D, Ang J M, Phua S L, Yee W A, Liu H, Huang Y, Lu X. Transition-metal-ion-mediated polymerization of dopamine: Mussel-inspired approach for the facile synthesis of robust transition-metal nanoparticlegraphene hybrids. Chem Eur J 20: 7776-7783 (2014)

[38] Ni Y, Chen Z, Kong F, Qiao Y, Kong A, Shan Y. Pony-size $\mathrm{Cu}$ nanoparticles confined in $\mathrm{N}$-doped mesoporous carbon by chemical vapor deposition for efficient oxygen electroreduction. Electrochim Acta 272: 233-241 (2018)

[39] Shi R, Zhao J, Liu S, Sun W, Li H, Hao P, Li Z, Ren J. Nitrogen-doped graphene supported copper catalysts for methanol oxidative carbonylation: Enhancement of catalytic activity and stability by nitrogen species. Carbon 130: 185-195 (2018)

of Solid Lubrication, Lanzhou Institute of Chemical Physics, Chinese Academy of Sciences. His research interests mainly focus on the tribology of composite materials.

designing functional surfaces with special wetting behavior, and engineering coatings for drag-reduction. He has published over 150 journal papers and gained a number of national scientific awards.

then he received his M.S. degree from Lanzhou Institute of Chemical Physics in 2009. His research areas mainly focus on the tribology of composite materials. 